\title{
Oceanic three-dimensional Lagrangian Coherent Structures: A study of a mesoscale eddy in the Benguela ocean region.
}

\author{
João H. Bettencourt ${ }^{1, *}$, Cristóbal López, Emilio Hernández-García \\ IFISC (CSIC-UIB), Instituto de Física Interdisciplinar y Sistemas Complejos \\ Campus Universitat de les Illes Balears \\ E-07122 Palma de Mallorca, Spain
}

\begin{abstract}
We study three dimensional oceanic Lagrangian Coherent Structures (LCSs) in the Benguela region, as obtained from an output of the ROMS model. To do that we first compute Finite-Size Lyapunov exponent (FSLE) fields in the region volume, characterizing mesoscale stirring and mixing. Average FSLE values show a general decreasing trend with depth, but there is a local maximum at about $100 \mathrm{~m}$ depth. LCSs are extracted as ridges of the calculated FSLE fields. They present a "curtain-like" geometry in which the strongest attracting and repelling structures appear as quasivertical surfaces. LCSs around a particular cyclonic eddy, pinched off from the upwelling front are also calculated. The LCSs are confirmed to provide pathways and barriers to transport in and out of the eddy.
\end{abstract}

Keywords: Lagrangian Coherent Structures, Finite-Size Lyapunov exponents, ocean transport, Benguela upwelling region, oceanic eddy

\section{Introduction.}

Mixing and transport processes are fundamental to determine the physical, chemical and biological properties of the oceans. From plankton dynamics to the evolution of pollutant spills, there is a wide range of practical issues that benefit from a correct understanding and modeling of these processes. Al- 32 though mixing and transport in the oceans occur in a wide range of scales, mesoscale and sub-mesoscale variability are known to play a very important role (Thomas et al., 2008; Klein and Lapeyre, 2009).

(11 Mesoscale eddies are especially important in this aspect be12. cause of their long life in oceanic flows, and their stirring and mixing properties. In the southern Benguela, for instance, cy-

${ }_{14}$ clonic eddies shed from the Agulhas current can transport and exchange warm waters from the Indian Ocean to the South Atlantic (Byrne et al., 1995; Lehahn et al., 2011). Moreover, mesoscale eddies have been shown to drive important biogeochemical processes in the ocean such as the vertical flux of nutrients into the euphotic zone (McGillicuddy et al., 1998, Oschlies and Garçon 1998). Another effect of eddy activity seems to be the intensification of mesoscale and sub-mesoscale variability due to the filamentation process where strong tracer gradients are created by the stretching of tracers in the shearand strain-dominated regions in between eddy cores (Elhmaïdi et al., 1993). Studies of the vertical structure of such eddies in the Benguela region (e. g. Doglioli et al. (2007) and Rubio

${ }^{*}$ Corresponding author Email address: joaob@ifisc.uib-csic.es (João H. Bettencourt) Phone: +34 971259905
27 et al. (2009)) have shown that they can extended to one thou8 sand meters deep waters.

In the last decades new developments in the description and modelling of oceanic mixing and transport from a Lagrangian viewpoint have emerged (Mariano et al., 2002, Lacasce, 2008). These Lagrangian approaches have become more and more frequent due to the increased availability of detailed knowledge of the velocity field from Lagrangian drifters, satellite measurements and computer models. In particular, the very relevant concept of Lagrangian Coherent Structure (LCS) (Haller. 2000, Haller and Yuan, 2000) is becoming crucial for the analysis of transport in flows. LCSs are structures that separate regions of the flow with different dynamical behavior. They give a general geometric view of the dynamics, acting as a (time-dependent) roadmap for the flow. They are templates serving as proxies to, for instance, barriers and avenues to transport or eddy boundaries (Boffetta et al., 2001; Haller and Yuan, 2000; Haller, 2002, d'Ovidio et al. 2004, 2009, Mancho et al., 2006).

The relevance of the three-dimensional structure of LCSs begins to be unveiled in atmospheric contexts (du Toit and Marsden, 2010, Tang et al., 2011, Tallapragada et al., 2011). In the case of oceanic flows, however, the identification of the LCSs and the study of their role on biogeochemical tracers transport has been mostly restricted to the marine surface (d'Ovidio et al. 2004; Waugh et al., 2006; d'Ovidio et al., 2009; Beron-Vera et al. 2008). This is mainly due to two reasons: a) tracer vertical displacement is usually very small with respect to the horizontal one; and b) satellite data of any quantity (temperature, chlorophyll, altimetry for velocity, etc..) are only available from the observation of the ocean surface. 
et al. (2011), applying the methodology of lobe dynamics and the turnstile mechanism to eddies pinched off from the Loop Current. In this paper we focus on FSLE fields and the associated particle trajectories to study transport in and out of the chosen mesoscale eddy. Since this is a first attempt to study $3 \mathrm{~d}$ oceanic LCS, more general results (on Benguela and other upwelling regions) are left for future work.

To circumvent the lack of appropriate observational data in the vertical direction, we use velocity fields from a numerical simulation. They are high resolution simulations from the ROMS model (see section 2 below) thus appropriate to study regional-medium scale basins.

The paper is organized as follows: In section II we describe the data and methods. In section III we present our results. Section IV contains a discussion of the results and Section V summarizes our conclusions.

\section{Data and Methods.}

\subsection{Velocity data set.}

The Benguela ocean region is situated off the west coast of southern Africa. It is characterized by a vigorous coastal upwelling regime forced by equatorward winds, a substantial mesoscale activity of the upwelling front in the form of eddies and filaments, and also by the northward drift of Agulhas eddies.

The velocity data set comes from a regional ocean model simulation of the Benguela Region (Le Vu et al., 2011). ROMS (Shchepetkin and McWilliams, 2003, 2005) is a split-explicit free-surface, topography following model. It solves the incompressible primitive equations using the Boussinesq and hydrostatic approximations. Potential temperature and salinity transport are included by coupling advection/diffusion schemes for these variables. The model was forced with climatological data. The data set area extends from $12^{\circ} \mathrm{S}$ to $35^{\circ} \mathrm{S}$ and from $4^{\circ} \mathrm{E}$ to $19^{\circ} \mathrm{E}$ (see Fig. 1). The velocity field $\mathbf{u}=(u, v, w)$ consists of two years of daily averaged zonal $(u)$, meridional $(v)$, and vertical velocity $(w)$ components, stored in a three-dimensional grid with an horizontal resolution of $1 / 12$ degrees $\sim 8 \mathrm{~km}$, and 32 vertical terrain-following levels using a stretched vertical coordinate where the layer thickness varies, increasing from the surface to the ocean interior. Since the ROMS model considers the hydrostatic approximation it is important to note that Mahadevan (2006), when comparing results from non-hydrostatic and hydrostatic versions of the same model of vertical motions at submesoscale fronts, found that while instantaneous vertical velocities structures differ, the averaged vertical flux is similar in both hydrostatic and non-hydrostatic simulations.

\subsection{Finite-Size Lyapunov Exponents.}

In order to study non-asymptotic dispersion processes such as stretching at finite scales and time intervals, the Finite Size Lyapunov Exponent (Aurell et al., 1997, Artale et al., 1997) is particularly well suited. It is defined as:

$$
\lambda=\frac{1}{\tau} \log \frac{\delta_{f}}{\delta_{0}},
$$




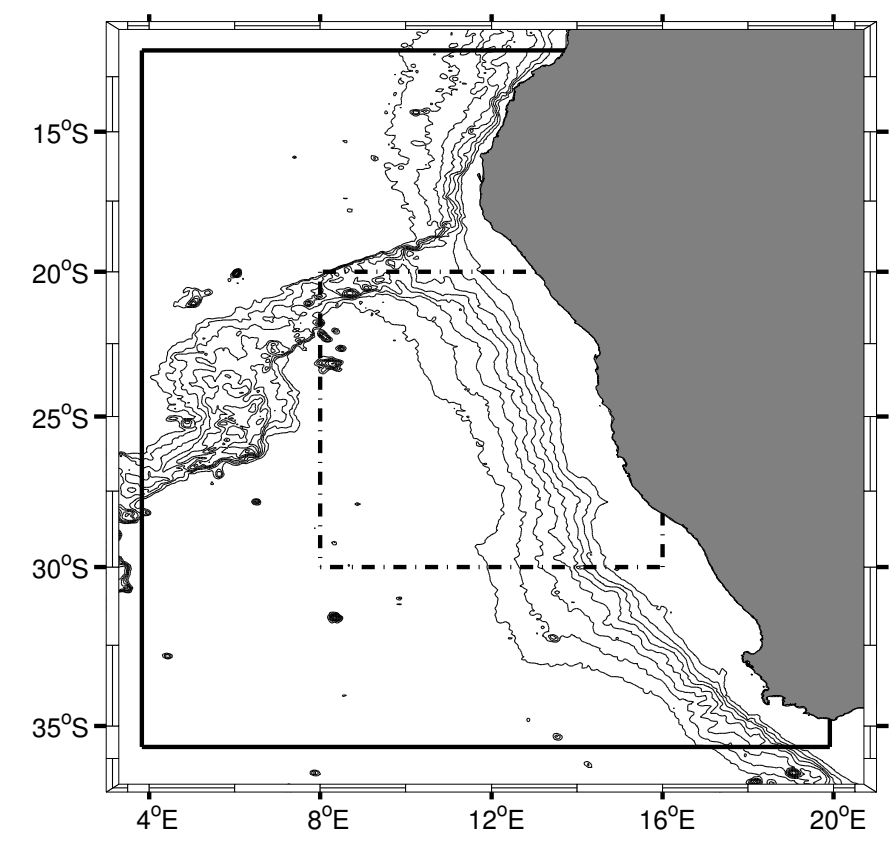

Figure 1: Benguela ocean region. The velocity field domain is limited by the continuous black line. The FSLE calculation area is limited by the dash-dot 215 black line. Bathymetric contour lines are from ETOPO1 global relief model 216 (Amante and Eakins 2009) starting a $0 \mathrm{~m}$ depth up to $4000 \mathrm{~m}$ at $500 \mathrm{~m}$ interval. 2 ticles, initially $\delta_{0}$, to reach $\delta_{f}$. In addition to the dependence on the values of $\delta_{0}$ and $\delta_{f}$, the FSLE depends also on the initial ${ }^{220}$ position of the particles and on the time of deployment. Lo- ${ }^{221}$ cations (i.e. initial positions) leading to high values of this ${ }^{222}$ Lyapunov field identify regions of strong separation between ${ }^{223}$ particles, i.e., regions that will exhibit strong stretching during ${ }^{224}$ evolution, that can be identified with the LCS (Boffetta et al. 2001; d'Ovidio et al. 2004, Joseph and Legras, 2002).

In principle, for computing FSLEs in three dimensions one just needs to extend the method of d'Ovidio et al. (2004), that is, one needs to compute the time that fluid particles initially separated by $\delta_{0}=\left[\left(\delta x_{0}\right)^{2}+\left(\delta y_{0}\right)^{2}+\left(\delta z_{0}\right)^{2}\right]^{1 / 2}$ need to reach a final distance of $\delta_{f}=\left[\left(\delta x_{f}\right)^{2}+\left(\delta y_{f}\right)^{2}+\left(\delta z_{f}\right)^{2}\right]^{1 / 2}$. The main difficulty in doing this is that in the ocean vertical displacements (even in upwelling regions) are much smaller than the horizontal ones, and so do not contribute significantly to total particle dispersion (Özgökmen et al. 2011). By the time the horizontal particle dispersion has scales of tenths or hundreds of kilometers (typical mesoscale structures are studied using $\delta_{f} \approx 100 \mathrm{~km}$ (d'Ovidio et al. (2004)), particle dispersion in the vertical can have at most scales of hundreds of meters and usually less. This means that the vertical separation will not contribute significantly to the accumulated distance between particles. In addition, since length scales in the horizontal and vertical differ by several orders of magnitude, one faces the impossibility of assigning equal $\delta_{0}$ to the horizontal and vertical particle pairs. It should be noted however that these shortcomings arise from the different scales of length and time that characterize horizontal and vertical dispersion processes in the ocean, and so should not be seem as intrinsic limitations of the method. For non-oceanic flows a di- ${ }_{247}$ where $\tau$ is the time it takes for the separation between two par- rect generalization of FSLEs is straightforward.

Thus, in this paper we implemented a quasi threedimensional computation of FSLEs. That is, we make the computation for every (2d) ocean layer, but where the particle trajectories calculation use the full $3 \mathrm{~d}$ velocity field. I.e., at each level (depth) we set $\delta z_{O}=0$, and the final distance is computed without taking the vertical distance between particles. It is important to note that, since we allow the particles to evolve in the full $3 \mathrm{~d}$ velocity field, we take into account vertical quantities such as vertical velocity shear that may influence the horizontal separation between particle pairs.

There are other possible approaches to the issue of different scales in the vertical and horizontal. One way is to assign anisotropic initial and final displacements in the FSLE calculation (i. e., including a $\delta z_{0}$ and $\delta z_{f}$ much smaller than the horizontal initial and final separations). A second approach is to use different weights for the horizontal and vertical separations in the calculations of the distance, perhaps in combination with the first. We have cheked both alternatives and found that, with reasonable choices of initial and final distances and distance metrics, the results were equivalent to the quasi-3d computation. The reason is that actual dispersion is primarily horizontal as commented above.

More in detail, a grid of initial locations $\mathbf{x}_{0}$ in the longitude/latitude/depth geographical space $(\phi, \theta, z)$, fixing the spatial resolution of the FSLE field, is set up at time $t$. The horizontal distance among the grid points, $\delta_{0}$, was set to $1 / 36$ degrees $(\approx 3 \mathrm{~km})$, i.e. three times finer resolution than the velocity field (Hernandez-Carrasco et al., 2011), and the vertical resolution (distance between layers) was set to $20 \mathrm{~m}$ in order to have a good representation of the vertical variations in the FSLE field. Particles are released from each grid point and their three dimensional trajectories calculated. The distances of each particle with respect to the ones that were initially neighbors at an horizontal distance $\delta_{0}$ are monitored until one of the horizontal separations reaches a value $\delta_{f}$. By integrating the three dimensional particle trajectories backward and forward in time, we obtain the two different types of FSLE maps: the attracting LCS (for the backward), and the repelling LCS (forward) (d'Ovidio et al., 2004; Joseph and Legras, 2002). We obtain in this way FSLE fields with a horizontal spatial resolution given by $\delta_{0}$. The final distance $\delta_{f}$ was set to $100 \mathrm{~km}$, which is, as already mentioned, a typical length scale for mesoscale studies. The trajectories were integrated for a maximum of $T=178$ days (approximately six months) using an integration time step of 6 hours. When a particle reached the coast or left the velocity field domain, the FSLE value at its initial position and initial time was set to zero. If the interparticle horizontal separation remains smaller than $\delta_{f}$ during all the integration time, then the FSLE for that location is also set to zero.

The equations of motion that describe the evolution of parti- 


\section{2.3. Lagrangian Coherent Structures.}

cle trajectories are

$$
\begin{aligned}
\frac{d \phi}{d t} & =\frac{1}{R_{z}} \frac{u(\phi, \theta, z, t)}{\cos (\theta)} \\
\frac{d \theta}{d t} & =\frac{1}{R_{z}} v(\phi, \theta, z, t), \\
\frac{d z}{d t} & =w(\phi, \theta, z, t),
\end{aligned}
$$
regular cube coordinate system. bolic lines. where $\phi$ is longitude, $\theta$ is latitude and $z$ is the depth. $R_{z}$ is the ${ }^{307}$ radial coordinate of the moving particle $R_{z}=R-z$, with $R={ }^{308}$ $6371 \mathrm{~km}$ the mean Earth radius. For all practical purposes, $R_{z} \approx{ }^{309}$ $R$. Particle trajectories are integrated using a $4^{\text {th }}$ order Runge- ${ }^{310}$ Kutta method. For the calculations, one needs the (3d) velocity ${ }^{311}$ values at the current location of the particle. Since the six grid 312 nodes surrounding the particle do not form a regular cube, direct ${ }_{313}$ trilinear interpolation can not be used. Thus, an isoparametric element formulation is used to map the nodes of the velocity grid surrounding the particles position to a regular cube, and ${ }^{315}$ an inverse isoparametric mapping scheme (Yuan et al., 1994) is used to find the coordinates of the interpolation point in the

In $2 \mathrm{~d}$, LCS practically coincide with (finite-time) stable and ${ }_{321}$ unstable manifolds of relevant hyperbolic structures in the flow ${ }_{222}$ (Haller, 2000, Haller and Yuan, 2000, Joseph and Legras, 323 2002). The structure of these last objects in $3 \mathrm{~d}$ is generally much more complex than in 2d (Haller, 2001, Pouransari et al. ${ }^{324}$ 2010), and they can be locally either lines or surfaces. As com- 325 mented before, however, vertical motions in the ocean are slow. 326 Thus, at each fluid parcel the strongest attracting and repelling ${ }_{327}$ directions should be nearly horizontal. This, combined with the incompressibility property, implies that the most attracting ${ }^{328}$ and repelling regions (i.e. the LCSs) should appear as almost ${ }_{329}$ vertical surfaces, since the attraction or repulsion should occur $3 з$ normally to the LCS. As a consequence, the LCSs will have a 331 "curtain-like" geometry, with deviations from the vertical due ${ }_{332}$ to either the orientation of the most attracting or repelling direction deviating from the horizontal, or when strong vertical shear ${ }_{334}$ produces variations along the vertical in the most repelling or ${ }_{335}$ attracting regions in the flow. We expect the LCS sheet-like ob- 336 jects to coincide with the strongest hyperbolic manifolds when ${ }_{33}$ these are two dimensional, and to contain the strongest hyper- $3 з 8$

The curtain-like geometry of the LCS was already com- 340 mented in Branicki and Malek-Madani (2010), Branicki and ${ }_{341}$ Kirwan (2010), or Branicki et al. (2011). In the latter paper 342 it was shown that, in a $3 \mathrm{~d}$ flow, these structures would appear ${ }_{343}$ mostly vertical when the ratio of vertical shear of the horizon- 344 tal velocity components to the average horizontal velocities is 345 small. This ratio also determines the vertical extension of the 346 structures. In Branicki and Kirwan (2010), the argument was 347 used to construct a 3d picture of hyperbolic structures from the 348 computation in a $2 \mathrm{~d}$ slice. In the present paper we confirm the 349 curtain-like geometry of the LCSs, and show that they are rele- 350 vant to organize the fluid flow in this realistic $3 \mathrm{~d}$ oceanic setting. 351
This is done in the next section by comparing actual particle trajectories with the computed LCSs.

Differently than $2 \mathrm{~d}$, where LCS can be visually identified as the maxima of the FSLE field, in $3 d$ the ridges are hidden within the volume data. Thus, one needs to explicitly compute and extract them, using the definition of LCSs as the ridges of the FSLEs. A ridge $L$ is a co-dimension 1 orientable, differentiable manifold (which means that for a three-dimensional domain $D$, ridges are surfaces) satisfying the following conditions (Lekien et al., 2007):

1. The field $\lambda$ attains a local extremum at $L$.

2. The direction perpendicular to the ridge is the direction of fastest descent of $\lambda$ at $L$.

Mathematically, the two previous requirements can be expressed as

$$
\begin{aligned}
\mathbf{n}^{\mathrm{T}} \nabla \lambda & =0, \\
\mathbf{n}^{\mathrm{T}} \mathbf{H n}=\min _{\|\mathbf{u}\|=1} \mathbf{u}^{\mathrm{T}} \mathbf{H u} & <0,
\end{aligned}
$$

where $\nabla \lambda$ is the gradient of the FSLE field $\lambda, \mathbf{n}$ is the unit normal vector to $L$ and $\mathbf{H}$ is the Hessian matrix of $\lambda$.

The method used to extract the ridges from the scalar field $\lambda\left(\mathbf{x}_{0}, t\right)$ is from Schultz et al. (2010). It uses an earlier (Eberly et al., 1994) definition of ridge in the context of image analysis, as a generalized local maxima of scalar fields. For a scalar field $f: \mathbb{R}^{n} \rightarrow \mathbb{R}$ with gradient $\mathbf{g}=\nabla f$ and Hessian $\mathbf{H}$, a $d$-dimensional height ridge is given by the conditions

$$
\forall_{d<i \leq n} \quad \mathbf{g}^{\mathrm{T}} \mathbf{e}_{i}=0 \text { and } \alpha_{i}<0,
$$

where $\alpha_{i}, i \in\{1,2, \ldots, n\}$, are the eigenvalues of $\mathbf{H}$, ordered such that $\alpha_{1} \geq \ldots \geq \alpha_{n}$, and $\mathbf{e}_{i}$ is the eigenvector of $\mathbf{H}$ associated with $\alpha_{i}$. For $n=3$, (7) becomes

$$
\mathbf{g}^{\mathrm{T}} \mathbf{e}_{3}=0 \text { and } \alpha_{3}<0 .
$$

This ridge definition is equivalent to the one given by (5) since the unit normal $\mathbf{n}$ is the eigenvector (when normalized) associated with the minimum eigenvalue of $\mathbf{H}$. In other words, in $\mathbb{R}^{3}$ the $\mathbf{e}_{1}, \mathbf{e}_{2}$ eigenvectors point locally along the ridge and the $\mathbf{e}_{3}$ eigenvector is orthogonal to it.

The ridges extracted from the backward FSLE map approximate the attracting LCS, and the ridges extracted from the forward FSLE map approximate the repelling LCS. The attracting ones are the more interesting from a physical point of view (d'Ovidio et al. 2004, 2009), since particles (or any passive scalar driven by the flow) typically approach them and spread along them, giving rise to filament formation. In the extraction process it is necessary to specify a threshold $s$ for the ridge strength $\left|\alpha_{3}\right|$, so that ridge points whose value of $\alpha_{3}$ is lower (in absolute value) than $s$ are discarded from the extraction process. Since the ridges are constructed by triangulations of the set of extracted ridge points, the $s$ threshold greatly determines the size and shape of the extracted ridge, by filtering out regions of the ridge that have low strength. The reader is referred to Schultz et al. (2010) for details about the ridge extraction method. The height ridge definition has been used to extract LCS from FTLE fields in several works (see, among others, Sadlo and Peikert (2007)). 


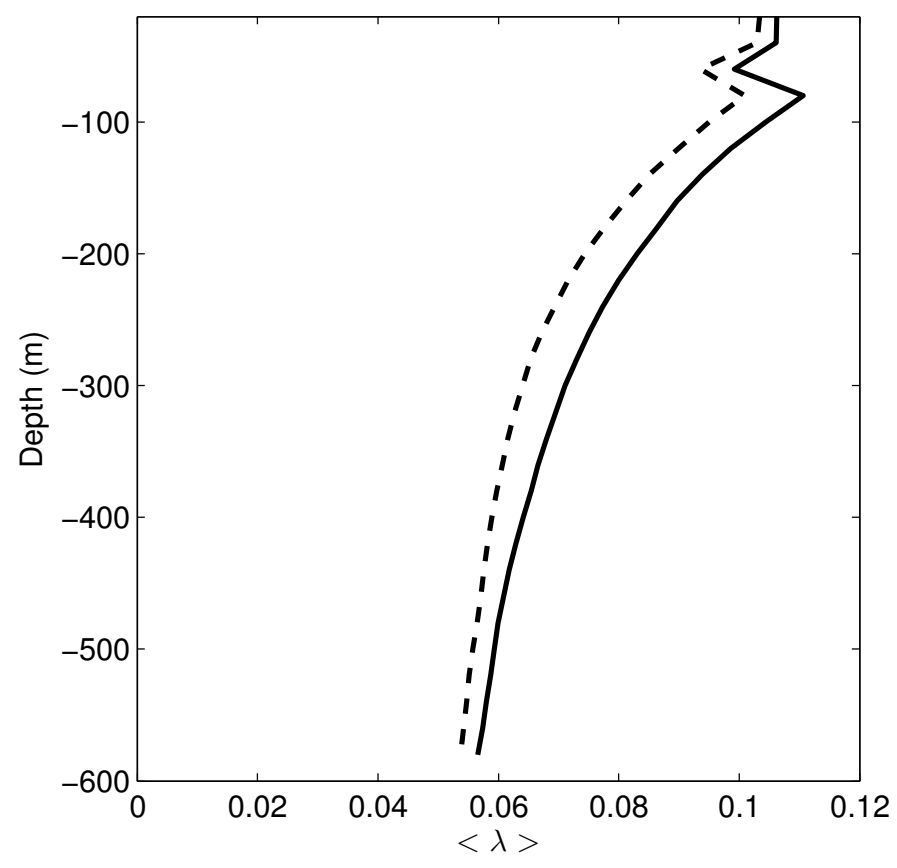

Figure 2: Vertical profile of 30 day average backward and forward FSLE. The 30 day average field was spatially averaged at each layer over the FSLE calculation area to produce the vertical profiles. The backward FSLE average is shown in continuous and the forward FSLE is shown in dashed.

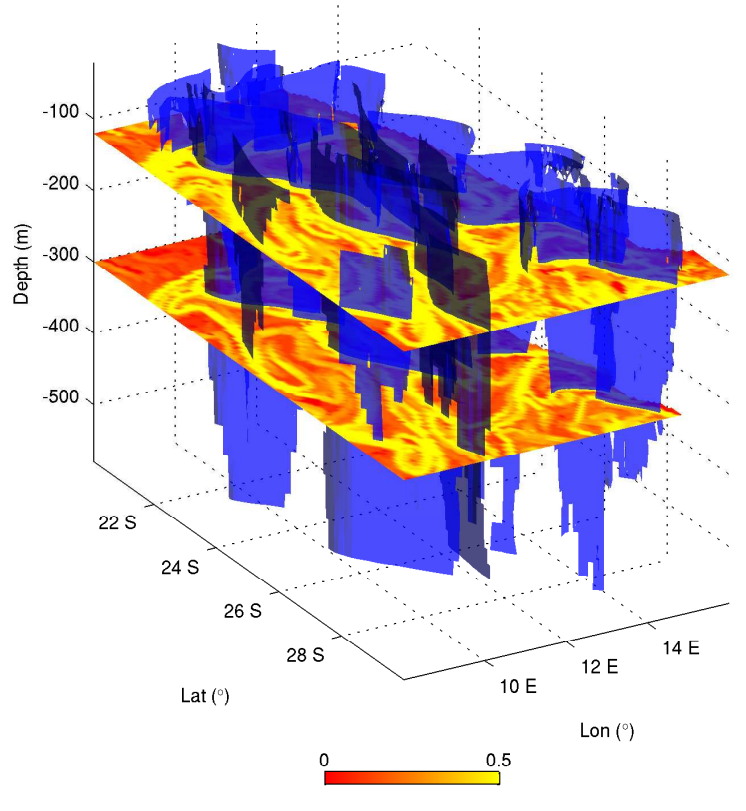

Figure 3: Attracting LCS (blue) for day 1 of the calculation period, together with horizontal slices of the backward FSLE field at $120 \mathrm{~m}$ and $300 \mathrm{~m}$ depth. Colorbar refers to colormap of horizontal slices. The units of the colorbar are $d a y^{-1}$.

The three dimensional FSLE field was calculated for a 30384 35 day period starting September 17, with snapshots taken every 385 2 days. The fields were calculated for an area of the Benguela 386 ocean region between latitudes $20^{\circ} \mathrm{S}$ and $30^{\circ} \mathrm{S}$ and longitudes ${ }_{387}$ $3588^{\circ} \mathrm{E}$ to $16^{\circ} \mathrm{E}$ (see figure 1 ). The area is bounded at NW by the 388 ${ }_{59}$ Walvis Ridge and the continental slope approximately bisects 389 60 the region from NW to SE. The western half of the domain has 390 abyssal depths of about $4000 \mathrm{~m}$. The calculation domain ex- 391 tended vertically from 20 up to $580 \mathrm{~m}$ of depth. Both backward 392 3 and forward calculations were made in order to extract the at- 393 tracting and repelling LCS.

Figure 2 displays the vertical profile of the average FSLE 395 ${ }_{66}$ for the 30 day period. There are small differences between the 396 backward and the forward values due to the different intervals 397 of time involved in their calculation. But both profiles have a 398 similar shape and show a general decrease with depth. There 399 is a notable peak in the profiles at about $100 \mathrm{~m}$ depth that indi- 400 cates increased mesoscale variability (and transport, as shown in Sect. 3.2 at that depth).

A snapshot of the attracting LCSs for day 1 of the calculation

period is shown in figure 3. As expected, the structures appear 402 as thin vertical curtains, most of them extending throughout the 403 depth of the calculation domain. The area is populated with 404 77 LCS, denoting the intense mesoscale activity in the Benguela 405 region. As already mentioned, in three dimensions the ridges 406 are not easily seen, since they are hidden in the volume data. 407 However the horizontal slices of the field in figure 3 show that 408 the attracting LCS fall on the maximum backward FSLE field 409 lines of the $2 \mathrm{~d}$ slices. The repelling LCS (not shown) also fall on the maximum forward FSLE field lines of the $2 d$ slices.

Since the $\lambda$ value of a point on the ridge and the ridges strength $\alpha_{3}$ are only related through the expressions (7) and (8), the relationship between the two quantities is not direct. This creates a difficulty in choosing the appropriate strength threshold for the extraction process. A too small value of $s$ will result in very small LCS that appear to have little influence on the dynamics, while a greater value will result in only a partial rendering of the LCS, limiting the possibility of observing their real impact on the flow. Computations with several values of $s$ lead us to the optimum choice $s=20 d a y^{-1} m^{-2}$, meaning that grid nodes with $\alpha_{3}<-20 d a y^{-1} m^{-2}$ were filtered out from the LCS triangulation.

We have seen in this section an example of how the ridges of the $3 d$ FSLE field, the LCS, distribute in the Benguela ocean region. Their ubiquity shows their impact on the transport and mixing properties. In the next section we concentrate on the properties of a single $3 \mathrm{~d}$ mesoscale eddy.

\subsection{Study of the dynamics of a relevant mesoscale eddy}

Let us study a prominent cyclonic eddy observed in the data set. The trajectory of the center of the eddy was tracked and it is shown in figure 4. The eddy was apparently pinched off at the upwelling front. At day 1 of the FSLE calculation period its center was located at latitude $24.8^{\circ} \mathrm{S}$ and longitude $10.6^{\circ} \mathrm{E}$, leaving the continental slope, and having a diameter of approximately $100 \mathrm{~km}$. One may ask: what is its vertical size? is it really a barrier, at any depth, for particle transport? 


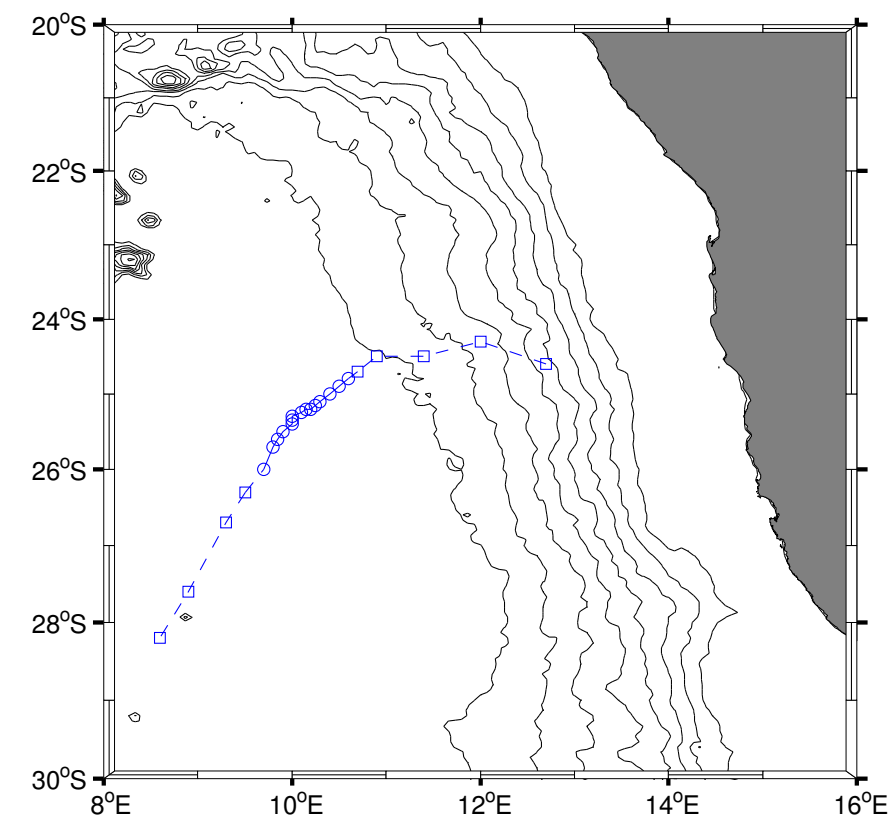

Figure 4: Trajectory (advancing from NE to SW) of the eddy center inside the 460 calculation domain. Circles indicate the center location during the 30 day FSLE 461 calculation period, and squares previous and posterior positions. Bathymetric lines same as in figure 1

To properly answer these questions the eddy, in particular its 46 411 frontiers, should be located. From the Eulerian point of view 466 412 it is commonly accepted that eddies are delimited by closed ${ }_{467}$ 413 contours of vorticity and that the existence of strong vorticity 468 414 gradients prevent the transport in and out of the eddy. Such 469 45 transport may occur when the eddy is destroyed or undergoes 470 strong interactions with other eddies (Provenzale, 1999). In a 471 Lagrangian view point, however, an eddy can be defined as a re- 472 48 gion delimited by intersections and tangencies of LCS, whether 473 in $2 \mathrm{~d}$ or $3 \mathrm{~d}$ space. The eddy itself is an elliptic structure ( 420 and Yuan, 2000, Branicki and Kirwan, 2010, Branicki et al., 475 421 2011). In this Lagrangian view of an eddy, the transport inhi- 476 422 bition to and from the eddy is now related to the existence of 477 423 these transport barriers delimiting the eddy region, which are 478 known to be quasi impermeable.

Using the first approach, i.e., the Eulerian view, the vertical 480 26 distribution of the $Q$-criteria (Hunt et al., 1988; Jeong and Hus- 481 22 Sain, 1995) was used to determine the vertical extension of the 482 428 mesoscale eddy. The $Q$ criterium is a $3 \mathrm{~d}$ version of the Okubo- ${ }_{483}$ Weiss criterium (Okubo, 1970; Weiss, 1991) and measures the 484 relative strength of vorticity and straining. In this context, ed- 485 dies are defined as regions with positive $Q$, with $Q$ the second ${ }_{486}$ invariant of the velocity gradient tensor

$$
Q=\frac{1}{2}\left(\|\mathbf{\Omega}\|^{2}-\|\mathbf{S}\|^{2}\right)
$$

(9) 4 the turnstile 490 Current, Branicki and Kirwan (2010) observed a net fluid enwhere $\|\mathbf{\Omega}\|^{2}=\operatorname{tr}\left(\mathbf{\Omega} \mathbf{\Omega}^{\mathrm{T}}\right),\|\mathbf{S}\|^{2}=\operatorname{tr}\left(\mathbf{S S}^{\mathrm{T}}\right)$ and $\mathbf{\Omega}, \mathbf{S}$ are the anti- 491 ${ }_{35}$ symmetric and symmetric components of $\nabla \mathbf{u}$. Using $Q=0$ as 492 436 the Eulerian eddy boundary, it can be seen from Fig. 5 that the 493 eddy extends vertically down to, at least, $600 \mathrm{~m}$.

Let us move to the Lagrangian description of eddies, which 495 ${ }_{439}$ is much in the spirit of our study, and will allow us to study 496 particle transport: eddies can be defined as the region bounded by intersecting or tangent repelling and attracting LCS (Branicki and Kirwan, 2010; Branicki et al., 2011). Using this criterion, and first looking at the surface located at $200 \mathrm{~m}$ depth, we see in Fig. 6 that certainly the Eulerian eddy seems to be located inside the area defined by several intersections and tangencies of the LCS. This eddy has an approximate diameter of $100 \mathrm{~km}$. In the south-north direction there are two intersections that appear to be hyperbolic points ( $\mathrm{H} 1$ and $\mathrm{H} 2$ in figure 6 ). In the West-East direction, the eddy is closed by a tangency at the western boundary, and a intersection of lines at the eastern boundary. The eddy core is devoid of high FSLE lines, indicating that weak stirring occurs inside (d'Ovidio et al., 2004). As additional Eulerian properties, we note that near or at the intersections $\mathrm{H} 1$ and $\mathrm{H} 2$ the $Q$-criterium indicates straining motions. In the case of $\mathrm{H} 2$, figure 5 (right panel) indicates high shear up to $200 \mathrm{~m}$ depth. The fact that the hyperbolic regions $\mathrm{H} 1$ and $\mathrm{H} 2$ lie in strain dominated regions of the flow $(Q<0)$ highlights the connection between hyperbolic particle behavior and instantaneous hyperbolic regions of the flow. The ridges of the FSLE field, however, do not remain in the negative $Q$ regions but cross into rotation dominated regions with $Q>0$. This indicates that there are some differences between the Eulerian view (Q) and the Lagrangian view (FSLE). It is the latter that can be understood in terms of particle behaviour as limiting regions of initial conditions (particles) that stay away from hyperbolic regions for long enough time (Haller and Yuan, 2000).

In $3 \mathrm{~d}$, the eddy is also surrounded by a set of attracting and repelling LCS (figure 7), calculated as explained in Subsection 2.3 . The lines identified in figure 6 are now seen to belong to the vertical of these surfaces.

Note that the vertical extent of these surfaces is in part determined by the strength parameter used in the LCS extraction process, so their true vertical extension is not clear from the grangian eddy boundary extends down to the maximum depth of the calculation domain, but moving northward it is seen that the LCS shorten their depth. Probably this does not mean that the eddy is shallower in the North, but rather that the LCS are losing strength (lower $\left|\alpha_{3}\right|$ ) and portions of it are filtered out by the extraction process. In any case, it is seen that as in two-dimensional calculations, the LCS delimiting the eddy do not perfectly coincide with its Eulerian boundary (Joseph and Legras, 2002), and we expect the Lagrangian view to be more relevant to address transport questions.

In the next paragraphs we analyze the fluid transport across the eddy boundary. Some previous results for Lagrangian eddies were obtained by Branicki and Kirwan (2010) and Branicki et al. (2011). Applying the methodology of lobe dynamics and trainment near the base of the eddy, and net detrainment near the surface, being fluid transport in and out of the eddy essentially confined to the boundary region. Let us see what happens in our setting.

We consider six sets of 1000 particles each, that were released at day 1 of the FSLE calculation period, and their trajecresults presented here. On the south, the closure of the La- 


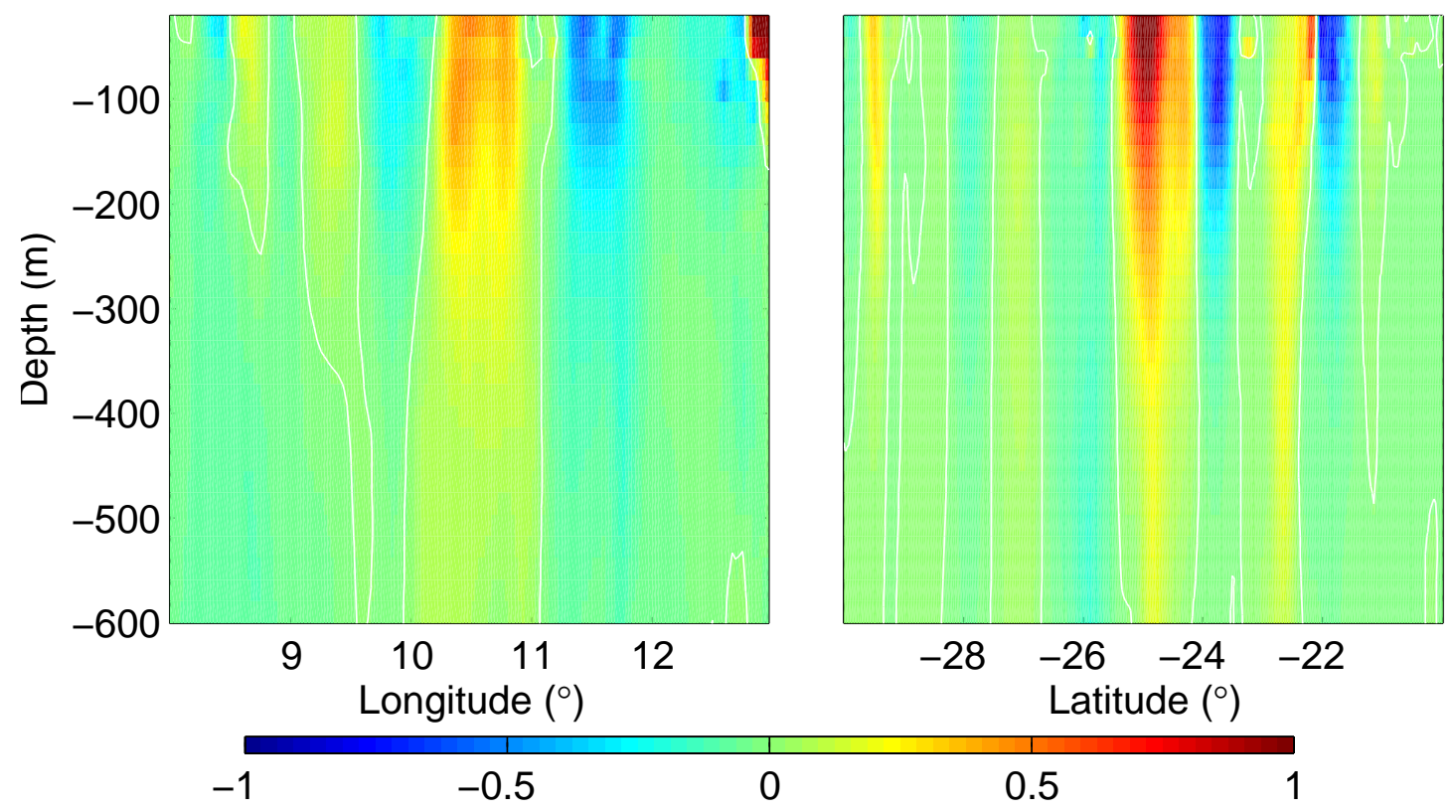

Figure 5: Colormap of $Q$-criterium. White contours have $Q=0$. Day 1 of the 30 day FSLE calculation period. Left panel: Latitude $24.5^{\circ} S$; Rigth panel: Longitude $10.5^{\circ} \mathrm{E}$. Colorbar values are $Q \times 10^{10} \mathrm{~s}^{-2}$.

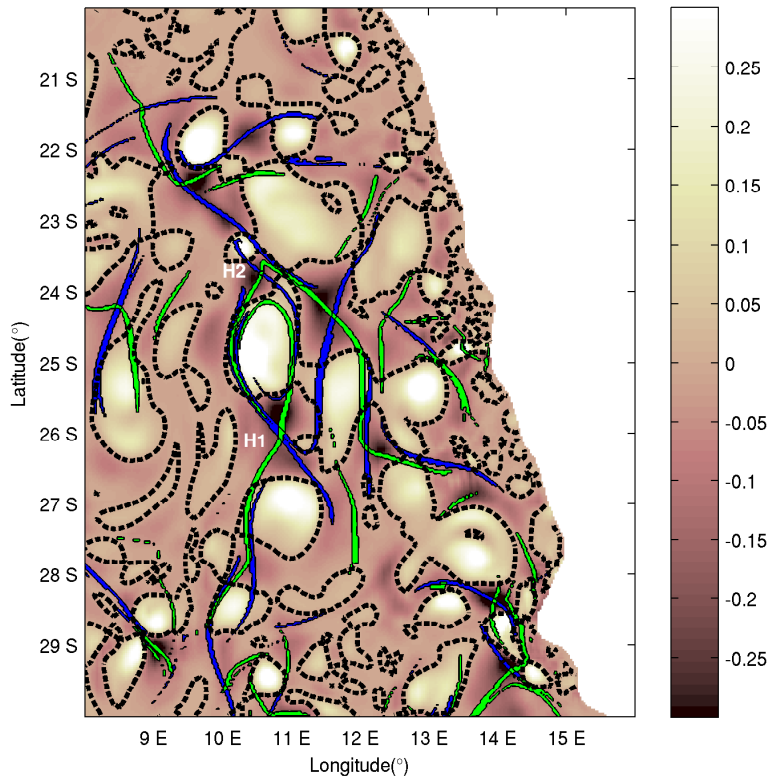

Figure 6: $Q$-criterium map at $200 \mathrm{~m}$ depth together with patches of backward (blue) and forward (green) FSLE values. Black dashed lines have $Q=0$. FSLE patches contain the highest $60 \%$ of FSLE values. Colorbar values are $Q \times$ $10^{10} s^{-2}$. The eddy we study is the clear region in between points $\mathrm{H} 1$ and $\mathrm{H} 2$.

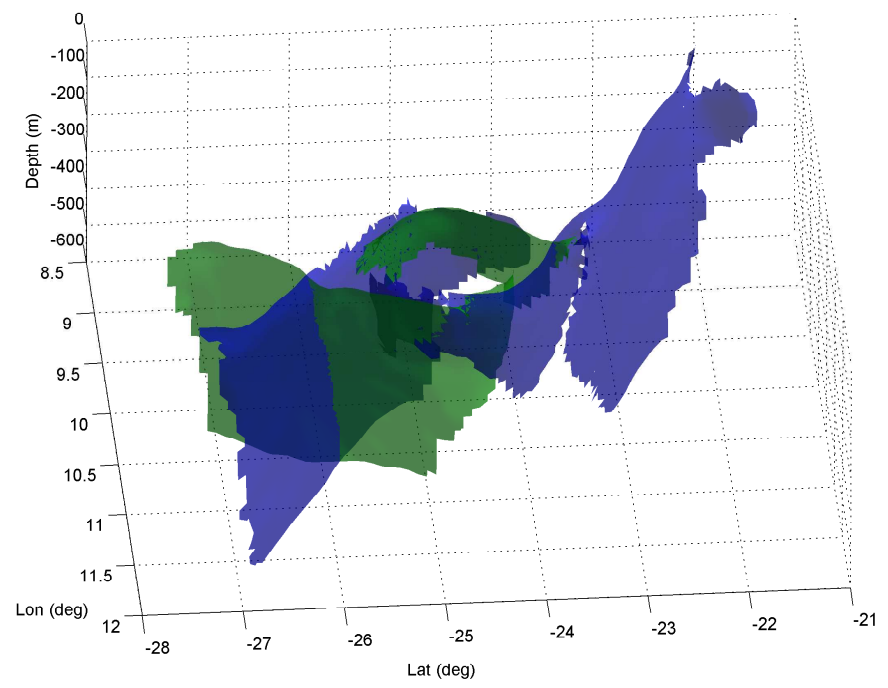

Figure 7: $3 \mathrm{~d}$ LCSs around the mesoscale eddy at day 1 of the 30 day FSLE calculation period. Green: repelling LCS; Blue: attracting LCS. 

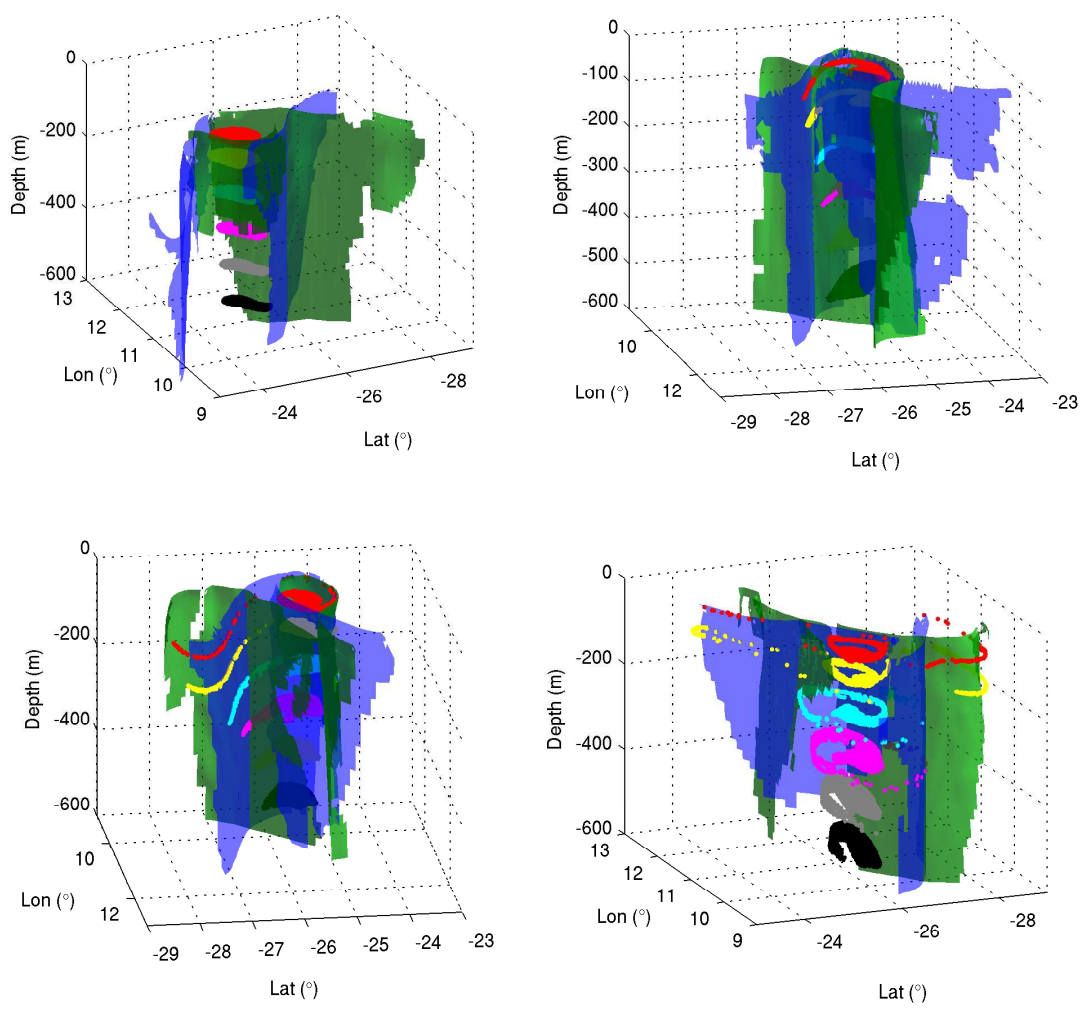

Figure 8: Three dimensional view of the evolution of elliptic patches released at different depths inside of the eddy at day 1 of the 30 day FSLE calculaton period. Top left: day 3; Top right: day 13; Bottom left: day 19: Bottom right: day 29. Red: 40 m; Yellow: 100 m; Cyan: 200 m; Magenta: 300 m; Grey: 400 m; Black: 500 $\mathrm{m}$. Attracting LCS are shaded in blue while repelling LCS are shaded in green.

tories integrated by a fourth-order Runge-Kutta method with a 521 integration time step of 6 hours. The sets of particles were re- 522 leased at depths of 50,100, 200,300, 400 and $500 \mathrm{~m}$. In figure 523 8 we plot the particle sets together with the Lagrangian bound- ${ }_{224}$ aries of the mesoscale eddy viewed in $3 \mathrm{~d}$. A top view is shown ${ }_{525}$ in figure 9. As expected, vertical displacements are small.

526

At day 3 (top left panel of figures 8 and 9 it can be seen that 527 there is a differential rotation (generally cyclonic, i.e. clock- 528 wise) between the sets of particles at different depths. The shal- 529 lower sets rotate faster than the deeper ones. This differential 530 rotation of the fluid particles could be viewed, in a Lagrangian 531 perspective, as the fact that the attracting and repelling strength ${ }_{32}$ of the LCS that limit the eddy varies with depth. Note that the ${ }^{53}$ six sets of particles are released at the same time and at the same 534 horizontal positions, and thereby their different behavior is due 535 to the variations of the LCS properties along depth.

At day 13 the vortex starts to expel material trough filamentation (Figs 8 and 9, top right panels). A fraction of the par- 538 ticles approach the southern boundaries of the eddy from the ${ }_{539}$ northeast. Those to the west of the repelling LCS (green) turn ${ }_{540}$ west and recirculate inside the eddy along the southern attract- 541 ing LCS (blue). Particles to the east of the repelling LCS turn ${ }_{542}$ east and leave the eddy forming a filament aligned with an at- ${ }_{543}$ tracting (blue) LCS. At longer times trajectories in the south of ${ }_{544}$ the eddy are influenced by additional structures associated to a different southern eddy. At day 29 (bottom right panels) the same process is seen to have occurred in the northern boundary, with a filament of particles leaving the eddy along the northern attracting (blue) LCS. The filamentation seems to begin earlier at shallower waters than at deeper ones since the length of the expelled filament diminishes with depth. However all of the expelled filaments follow the same attracting LCS. Figure 10 shows the stages previous to filamentation in which the LCS structure, their tangencies and crossings, and the paths of the particle patches are more clearly seen. Note that the LCS do not form fully closed structures and the particles escape the eddy through their openings. The images suggest lobe-dynamics processes, but much higher precision in the LCS extraction would be needed to really see such details.

This filamentation event seems to be the only responsible for transport of material outside of the eddy, since the rest of the particles remained inside the eddy boundaries. To get a rough estimate of the amount of matter expelled in the filamentation process we tracked the percentage of particles leaving a circle of diameter $200 \mathrm{~km}$ centered on the eddy center. In Fig. 11 the time evolution of this percentage is shown for the particle sets released at different depths. The onset of filamentation is clearly visible around days 9-12 as a sudden increase in the per- 

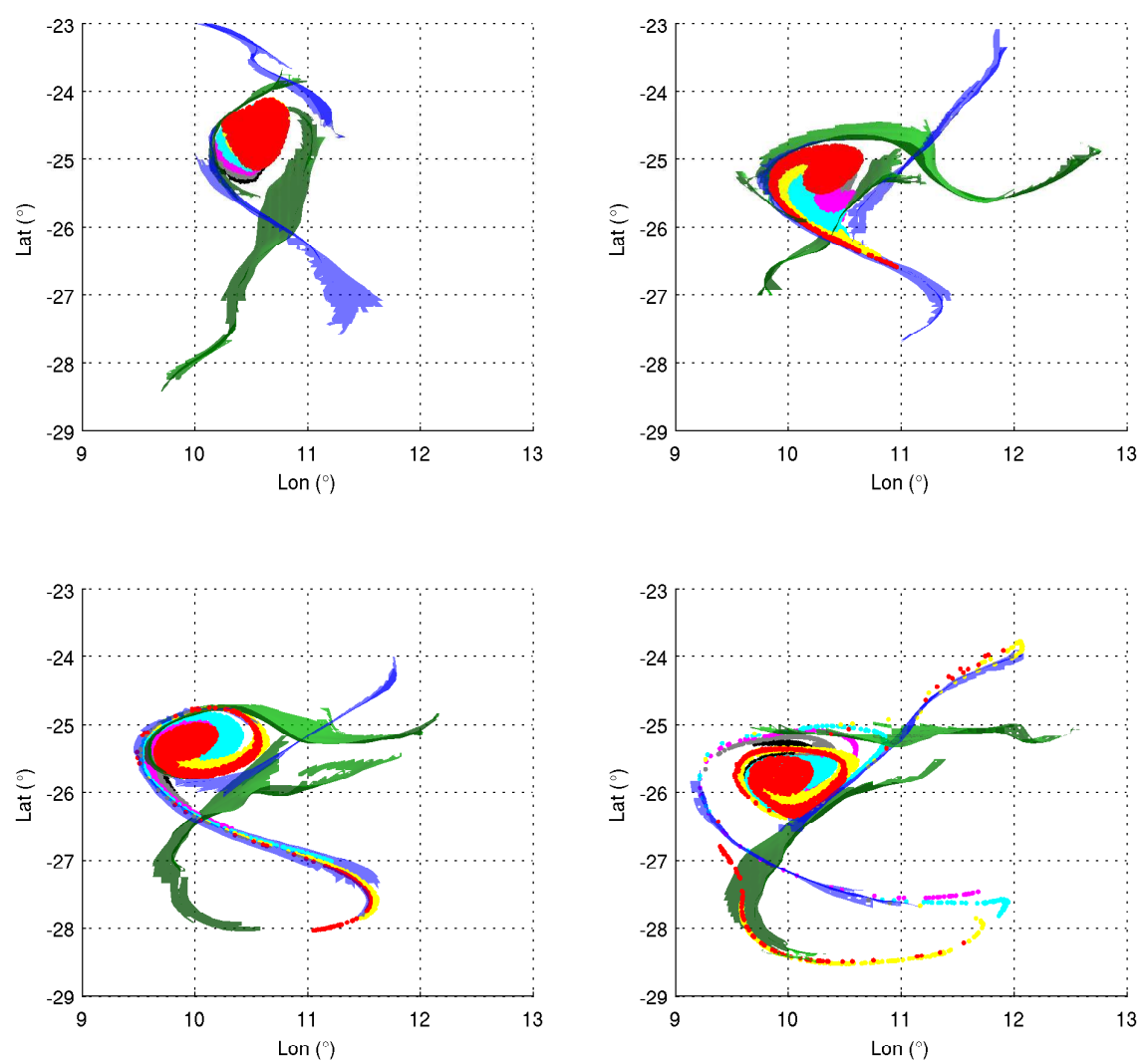

Figure 9: Top view of the evolution of particle patches and LCSs shown in Fig. 8. Top left: day 3; Top right: day 13; Bottom left: day 19: Bottom right: day 29. Colors as in figure 8 .

centage of particles leaving the eddy. The percentage is maxi- 566 mum for the particles located at $100 \mathrm{~m}$ depth and decreases as 567 the depth increases. At 400 and $500 \mathrm{~m}$ depth there are no parti- 568 cles leaving the circle. There is a clear lag between the onset of 569 filamentation between the different depths: the onset is simulta- 570 neous for the $40 \mathrm{~m}$ and $100 \mathrm{~m}$ depths but occurs later for larger 571 depths.

\section{Discussion.}

The spatial average of FSLEs defines a measure of stirring 576 and thus of horizontal mixing between the scales used for its 577 computation. The larger the average, the larger the mixing ac- 578 tivity (d'Ovidio et al. 2004). The general trend in the verti- 579 cal profiles of the average FSLE (Fig. 37 shows a reduction of 580 mesoscale mixing with depth. There is however a rather inter- 581 esting peak in this average profile occurring at $100 \mathrm{~m}$, i.e. close 582 to the thermocline. It could be related to submesoscale pro- ${ }_{583}$ cesses that occur alongside the mesoscale ones. Submesoscale 584 is associated to filamentation (the thickness of filaments is of 585 the order of $10 \mathrm{~km}$ or less), and we have seen that the filamen- 586 tation and the associated transport intensity (Fig. 11) is higher 587 at $100 \mathrm{~m}$ depth. It is not clear at the moment what is the precise 588 mechanism responsible for this increased activity at around 100 $m$ depth (perhaps associated to instabilities in the mixed layer), but we note that the intensity of shearing motions (see the $Q$ plots in 5) is higher in the top 200 meters. Less intense filamentation could be caused by reduction of shear in depths larger than these values.

From an Eulerian perspective, it is thought that vortex fil3 amentation occurs when the potential vorticity (PV) gradient 4 aligns itself with the compressional axis of the velocity field, in strain coordinates (Louazel and Hua (2004):Lapeyre et al. (1999)). This alignment is accompanied by exponential growth of the PV gradient magnitude. The fact that the filamentation occurs along the attracting LCS seems to indicate that this exponential growth of the PV gradient magnitude occurs across the attracting LCS.

In the specific spatiotemporal area we have studied, and in particular, for the eddy on which we focussed our analysis, we have confirmed that the structure of the LCSs is "curtain-like", so that the strongest attracting and repelling structures are quasivertical surfaces. Their vertical extension would depend of the physical transport properties, but it is also altered by the particular threshold parameter selected to extract the LCSs. These observations imply that transport and stirring occurs mainly on 

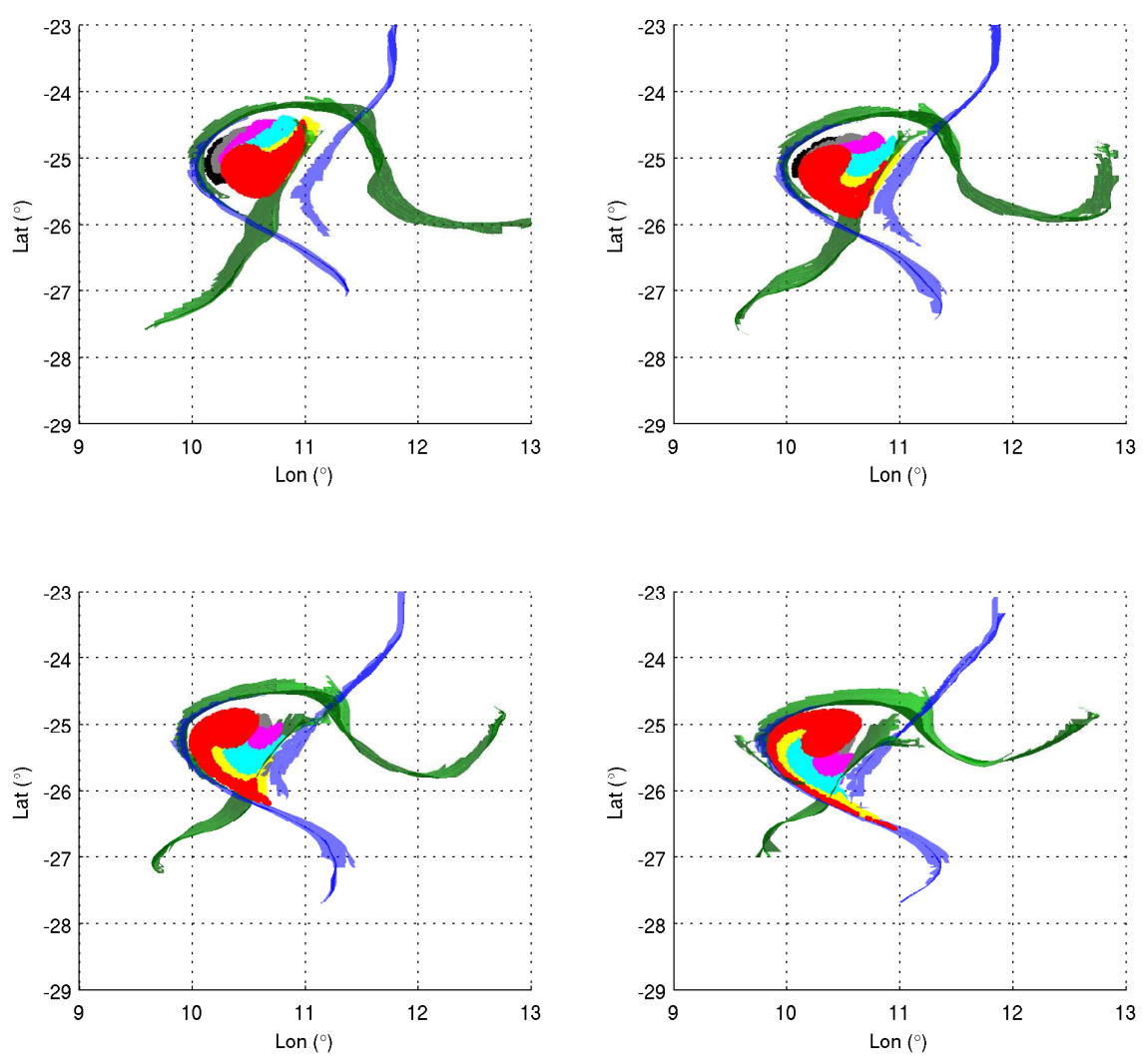

Figure 10: Top view of the initial stages of evolution of the particle patches and LCSs of Figs. 8 and 9. Top left: day 7; Top right: day 9; Bottom left: day 11: Bottom right: day 13. Colors as in figure 8 .

Another possible limitation worth mentioning is the velocity field resolution and its relation to the intensity of the vertical velocity. It is accepted that in fronts or in the eddy periphery, vertical velocities are significantly greater than, for instance, in the eddy interior. These zones of enhanced vertical transport correspond to submesoscale features that were not adequately captured in the velocity field used in this work due to its coarse resolution, since submesoscale studies usually have resolutions $<10 \mathrm{~km}$ (the literature on this subject is quite large, so we refer the reader to Klein and Lapeyre (2009) and Lévy (2008) ).

In any case, a most important point for the LCS we have computed is that in $3 \mathrm{~d}$, as in $2 \mathrm{~d}$, they act as pathways and barriers to transport, so that they provide a skeleton organizing the transport processes.

\section{Conclusions}

Three dimensional Lagrangian Coherent Structures were used to study stirring processes leading to dispersion and mixing at the mesoscale in the Benguela ocean region. We have computed 3d Finite Size Lyapunov Exponent fields, and LCSs were identified with the ridges these fields. LCSs appear as quasivertical surfaces, so that horizontal cuts of the FSLE fields 


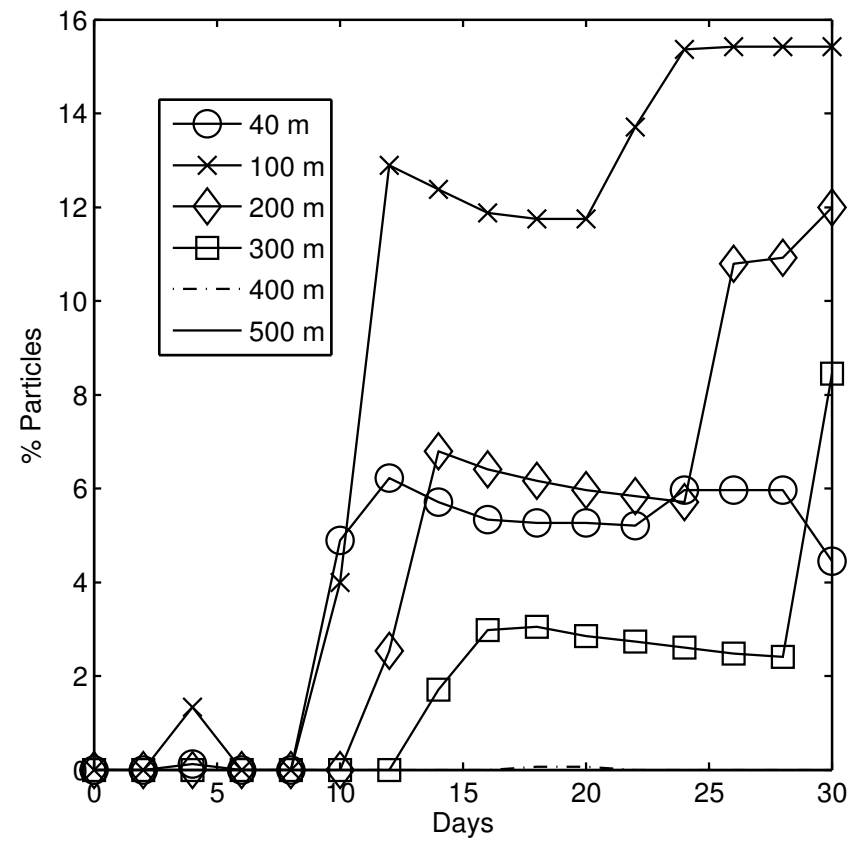

Figure 11: Percentage of particles outside a $200 \mathrm{~km}$ diameter circle centered at the eddy center, as a function of time. gives already a quite accurate vision of the 3d FSLE distribu- 686 tion. These quasivertical surfaces appear to be coincident with ${ }^{687}$ the maximal lines of the FSLE field (see fig. 3) so that sur- ${ }_{689}^{689}$ face FSLE maps could be indicative of the position of 3d LCS, 690 as long as the vertical shear of the velocity does not result in a ${ }^{691}$ significant deviation of the LCS with respect to the vertical. Average FSLE values generally decrease with depth, but we find ${ }_{69}^{69}$ a local maximum, and thus enhanced stretching and dispersion, 695 at about $100 \mathrm{~m}$ depth.

We have also analyzed a prominent cyclonic eddy, pinched ${ }_{698}^{697}$ off the upwelling front and study the filamentation dynamics 699 in 3d. Lagrangian boundaries of the eddy were made of in- 700 tersections and tangencies of attracting and repelling LCS that ${ }_{702}^{701}$ apparently emanating from two hyperbolic locations North and ${ }_{703}^{702}$ South of the eddy. The LCS are seen to provide pathways and 704 barriers organizing the transport processes and geometry. This ${ }^{705}$ pattern extends down up to the maximum depth were we cal- ${ }_{707}^{706}$ culated the FSLE fields $(\sim 600 \mathrm{~m})$, but the exact shape of the ${ }_{708}$ boundary is difficult to determine due to the decrease in ridge 709 strength with depth. This caused some parts of the LCS not to ${ }^{710}$ be extracted. The inclusion of a variable strength parameter in ${ }_{712}^{711}$ the extraction process is an important step to be included in the ${ }_{713}$ future.

The filamentation dynamics, and thus the transport out of the ${ }^{715}$ eddy, showed time lags with increasing depth. This arises from 717 the vertical variation of the flow field. However the filamenta- 718 tion occurred along all depths, indicating that in reality vertical ${ }_{720}^{719}$ sheets of material are expelled from these eddies.

Many more additional studies are needed to further clarify 722 the details of the geometry of the LCSs, their relationships with ${ }^{723}$ finite-time hyperbolic manifolds and three dimensional lobe dy- ${ }^{224}$ namics, and specially their interplay with mesoscale and sub- 726
${ }_{65}$ mesoscale transport and mixing processes.

\section{Acknowledgements}

Financial support from Spanish MICINN and FEDER through project FISICOS (FIS2007-60327) and from CSIC Intramural project TurBiD is acknowledged. JHB acknowledges financial support of the Portuguese FCT (Foundation for Science and Technology) through the predoctoral grant SFRH/BD/63840/2009. We thank the LEGOS group for providing us with $3 \mathrm{D}$ outputs of the velocity fields from their coupled BIOBUS/ROMS climatological simulation. The ridge extraction algorithm of Schultz et al. (2010) is available in the seek module of the data visualization library Teem (http://teem.sf.net).

\section{References}

Amante, C., Eakins, B.W., 2009. ETOPO1 1 Arc-Minute Global Relief Model: Procedures, Data Sources and Analysis. NOAA Technical Memorandum NESDIS NGDC-24.

Artale, V., Boffetta, G., Celani, A., Cencini, M., Vulpiani, A., 1997. Dispersion of passive tracers in closed basins: Beyond the diffusion coefficient. Phys. Fluids 9, 3162-3171

Aurell, E., Boffetta, G., Crisanti, A., Paladin, G., Vulpiani, A., 1997. Predictability in the large: an extension of the Lyapunov exponent. J. Phys. A $30,1-26$.

Beron-Vera, F., Olascoaga, M., Goni, G., 2008. Oceanic mesoscale eddies as revealed by Lagrangian coherent structures. Geophys. Res. Lett 35, L12603.

Boffetta, G., Lacorata, G., Redaelli, G., Vulpiani, A., 2001. Detecting barriers to transport: a review of different techniques. Physica D 159, 58-70.

Branicki, M., Kirwan, A., 2010. Stirring: The Eckart paradigm revisited. Int. J. Eng. Sci. 48, 1027-1042.

Branicki, M., Malek-Madani, R., 2010. Lagrangian structure of flows in the Chesapeake Bay: challenges and perspectives on the analysis of estuarine flows. Nonlinear Processes in Geophysics 17, 149-168.

Branicki, M., Mancho, A.M., Wiggins, S., 2011. A Lagrangian description of transport associated with a front-eddy interaction: Application to data from the North-Western Mediterranean sea. Physica D: Nonlinear Phenomena 240, $282-304$.

Branicki, M., Wiggins, S., 2009. Finite-time Lagrangian transport analysis: Stable and unstable manifolds of hyperbolic trajectories and finite-time Lyapunov exponents. Nonlinear Processes in Geophysics 17, 1-36.

Byrne, D.A., Gordon, A.L., Haxby, W.F., 1995. Agulhas eddies: A synoptic view using Geosat ERM data. J. Phys. Oceanogr. 25, 902-917.

Doglioli, A., Blanke, B., Speich, S., Lapeyre, G., 2007. Tracking coherent structures in a regional ocean model with wavelet analysis: Application to cape basin eddies. Journal of Geophysical Research C: Oceans 112, C05043.

d'Ovidio, F., Fernández, V., Hernández-García, E., López, C., 2004. Mixing structures in the Mediterranean sea from Finite-Size Lyapunov Exponents. Geophys. Res. Lett. 31, L17203.

d'Ovidio, F., Isern, J., López, C., Hernández-García, E., García-Ladona, E., 2009. Comparison between Eulerian diagnostics and Finite-Size Lyapunov Exponents computed from Altimetry in the Algerian basin. Deep-Sea Res. I 56, 15-31.

Eberly, D., Gardner, R., Morse, B., Pizer, S., Scharlach, C., 1994. Ridges for image analysis. Journal of Mathematical Imaging and Vision 4, 353-373.

Elhmaïdi, D., Provenzale, A., Babiano, A., 1993. Elementary topology of twodimensional turbulence from a Lagrangian viewpoint and single-particle dispersion. J. Fluid Mech. 257, 533-558.

Haller, G., 2000. Finding finite-time invariant manifolds in two-dimensional velocity fields. Chaos 10(1), 99-108.

Haller, G., 2001. Distinguished material surfaces and coherent structure in three-dimensional fluid flows. Physica D 149, 248-277.

Haller, G., 2002. Lagrangian coherent structures from approximate velocity data. Phys. Fluids A14, 1851-1861. 
727 Haller, G., 2011. A variational theory of hyperbolic lagrangian coherent struc- 798 tures. Physica D 240, 574-598.

729 Haller, G., Yuan, G., 2000. Lagrangian coherent structures and mixing in two- 800 dimensional turbulence. Physica D 147, 352-370.

Haza, A., Özgökmen, T., Griffa, A., Molcard, A., Poulain, P.M., Peggion, G., 802 2010. Transport properties in small-scale coastal flows: relative dispersion 803 from VHF radar measurements in the Gulf of La Spezia. Ocean Dynamics 804 $60,861-882$.

Haza, A.C., Poje, A.C., Özgökmen, T.M., Martin, P., 2008. Relative dispersion 806 from a high-resolution coastal model of the Adriatic Sea. Ocean Modell. 22, 807 48-65.

Hernandez-Carrasco, I., López, C., Hernández-García, E., Turiel, A., 2011. 809 How reliable are finite-size Lyapunov exponents for the assessment of ocean 810 dynamics? Ocean Modell. 36, 208-218.

Hunt, J.C.R., Wray, A.A., Moin, P., 1988. Eddies, Streams and Convergence 812 Zones in Turbulent Flows. Technical Report CTR-S88. Center for Turbu- 813 lence Research, Standford University. 193-208.

Jeong, J., Hussain, F., 1995. On the identification of a vortex. J. Fluid Mech. 81 $285,69-94$.

Joseph, B., Legras, B., 2002. Relation between kinematic boundaries, stirring, 817 and barriers for the Antartic polar vortex. J. Atm. Sci. 59, 1198-1212. 818

Klein, P., Lapeyre, G., 2009. The oceanic vertical pump induced by mesoscale 819 and submesoscale turbulence. Ann. Rev. Mar. Sci. 1, 351-375.

Lacasce, J., 2008. Statistics from Lagrangian observations. Progress in 821 oceanography $77,1-29$.

Lapeyre, G., Klein, P., Hua, B., 1999. Does the tracer gradient vector align with 823 strain eigenvectors in 2d turbulence? Phys. Fluids 11, 3729-3737. 824

Le Vu, B., Gutknecht, E., Machu, E., Dadou, I., Veitch, J., Sudre, J., Paul- 825 mier, A., Garçon, V., 2011. Processes maintaining the OMZ of the benguela 826 upwelling systemusing an eddy resolving model. submitted to JMR .

Lehahn, Y., d'Ovidio, F., Lévy, M., Amitai, Y., Heifetz, E., 2011. Long range 828 transport of a quasi isolated chlorophyll patch by an Agulhas ring. Geophys. 829 Res. Lett. 38, L16610.

Lekien, F., Shadden, S.C., Marsden, J.E., 2007. Lagrangian coherent structures 83 in n-dimensional systems. J. Math. Phys. 48, 065404.

Louazel, S., Hua, B.L., 2004. Vortex erosion in a shallow-water model. Physics 833 of Fluids 16, 3079-3085.

Lévy, M., 2008. The modulation of biological production by oceanic mesoscale 835 turbulence, in: Weiss, J., Provenzale, A. (Eds.), Transport and Mixing in 836 Geophysical Flows. Springer Berlin / Heidelberg. volume 744 of Lecture 837 Notes in Physics, pp. 219-261.

Mahadevan, A., 2006. Modeling vertical motion at ocean fronts: Are nonhy- 83 drostatic effects relevant at submesoscales? Ocean Modelling 14, 222 - 840 240.

Mancho, A.M., Small, D., Wiggins, S., 2006. A tutorial on dynamical systems 842 concepts applied to Lagrangian transport in ocean flows defined as finite time data sets: theoretical and computational issues. Phys. Rep. 437, 55124.

Mariano, A.J., Griffa, A., Özgökmen, T., Zambianchi, E., 2002. Lagrangian analysis and predictability of coastal and ocean dynamics 2000. J. Atmos. Oceanic Technol. 19, 1114-1126.

McGillicuddy, D.J., Robinson, A.R., Siegel, D.A., Jannasch, H.W., Johnson, R., Dickey, T.D., McNeil, J., Michaels, A.F., Knap, A.H., 1998. Influence of mesoscale eddies on new production in the Sargasso sea. Nature 394 263-266.

Molcard, A., Poje, A., Özgökmen, T., 2006. Directed drifter launch strategies for Lagrangian data assimilation using hyperbolic trajectories. Ocean Modell. $12,268-289$.

Okubo, A., 1970. Horitzontal dispersion of floatable particles in the vicinity of velocity singularities such as convergences. Deep-Sea Res. I 17, 445-454.

Oschlies, A., Garçon, V., 1998. Eddy-induced enhancement of primary productivity in a model of the North Atlantic ocean. Nature 394, 266-269.

Özgökmen, T.M., Poje, A.C., Fischer, P.F., Haza, A.C., 2011. Large eddy simulations of mixed layer instabilities and sampling strategies. Ocean Modell. 39, 311-331.

Pauly, D., Christensen, V., 1995. Primary production required to sustain global fisheries 374, 255-257.

Poje, A.C., Haza, A.C., Özgökmen, T.M., Magaldi, M.G., Garraffo, Z.D., 2010. Resolution dependent relative dispersion statistics in a hierarchy of ocean models. Ocean Modell. 31, 36-50.

Pouransari, Z., Speetjens, M., Clercx, H., 2010. Formation of coherent struc- tures by fluid inertia in three-dimensional laminar flows. J. Fluid Mech. 654, 5-34.

Provenzale, A., 1999. Transport by coherent barotropic vortices. Annu. Rev. Fluid Mech. 31, 55-93.

Rossi, V., López, C., Sudre, J., Hernández-García, E., Garçon, V., 2008. Comparative study of mixing and biological activity of the Benguela and Canary upwelling systems. Geophys. Res. Lett. 35, L11602.

Rubio, A., Blanke, B., Speich, S., Grima, N., Roy, C., 2009. Mesoscale eddy activity in the southern Benguela upwelling system from satellite altimetry and model data. Progress In Oceanography 83, $288-295$.

Sadlo, F., Peikert, R., 2007. Efficient visualization of Lagrangian Coherent Structures by filtered AMR ridge extraction. IEEE Transactions on Visualization and Computer Graphics 13, 1456-1463.

Schultz, T., Theisel, H., Seidel, H.P., 2010. Crease surfaces: From theory to extraction and application to diffusion tensor MRI. IEEE Transactions on Visualization and Computer Graphics 16, 109-119.

Shadden, S.C., Lekien, F., Marsden, J.E., 2005. Definition and properties of Lagrangian coherent structures from finite-time Lyapunov exponents in twodimensional aperiodic fows. Physica D. 212, 271-304.

Shchepetkin, A.F., McWilliams, J.C., 2003. A method for computing horizontal pressure-gradient force in an oceanic model with a nonaligned vertical coordinate. J. Geophys. Res. 108, 3090.

Shchepetkin, A.F., McWilliams, J.C., 2005. The regional ocean modeling system: A split-explicit, free-surface, topography following coordinates ocean model. Ocean Modell. 9, 347-404.

Tallapragada, P., Ross, S.D., Schmale III, D.G., 2011. Lagrangian coherent structures are associated with fluctuations in airborne microbial populations. Chaos 21, 033122

Tang, W., Chan, P.W., Haller, G., 2011. Lagrangian coherent structure analysis of terminal winds detected by lidar. part i: Turbulence structures. Journal of Applied Meteorology and Climatology 50, 325-338.

Thomas, L., Tandon, A., Mahadevan, A., 2008. Submesoscale ocean processes and dynamics, in: Hecht, M., Hasume, H. (Eds.), Ocean Modeling in an Eddying Regime, Geophysical Monograph 177, American Geophysical Union, Washington D.C.. pp. 17-38.

du Toit, P., Marsden, J., 2010. Horseshoes in hurricanes. Journal of Fixed Point Theory and Applications 7, 351-384. 10.1007/s11784-010-0028-6.

Waugh, D.W., Abraham, E.R., Bowen, M.M., 2006. Spatial variations of stirring in the surface ocean: A case of study of the Tasman sea. J. Phys. Oceanogr. 36, 526-542.

Weiss, J., 1991. The dynamics of enstrophy transfer in two-dimensional hydrodynamics. Physica D 48, 273-294.

Yuan, K.Y., Huang, Y.S., Yang, H.T., Pian, T.H.H., 1994. The inverse mapping and distortion measures for 8-node hexahedral isoparametric elements. Computational Mechanics 14, 189-199. 